\title{
Effect of Phonon Anomalies on the Shear Response of Martensitic Crystals
}

\author{
Y. Y. Ye, (a) C. T. Chan, and K. M. Ho \\ Ames Laboratory-Department of Energy and Department of Physics, Iowa State University, Ames, Iowa 50011
}

(Received 26 April 1990; revised manuscript received 16 January 1991)

\begin{abstract}
The shear response of a prototypical martensitic system is studied using a simple model incorporating the effects of both anharmonicity and anomalous phonon behavior. Using parameters determined from experimental phonon data, we show that the pseudoelastic and thermoelastic effects characteristic of $\mathrm{Ni}-\mathrm{Al}$ and many other shape-memory alloys are closely related to the observed phonon anomalies.
\end{abstract}

PACS numbers: $62.20 . \mathrm{Fe}, 63.20 . \mathrm{Dj}, 81.30 . \mathrm{Kf}$

The subject of martensitic phase transformation is one of considerable scientific as well as technological interest. Among the martensitic materials, the "shape-memory" alloys have received special attention. ${ }^{1,2}$ These alloys exhibit curious elastic behavior near the martensitic transformation temperature $\left(M_{s}\right)$ : They can be strained beyond the elastic limit in a reversible manner. ${ }^{3}$ In some cases, the return to the initial state occurs when the loading stress is released (pseudoelastic effect) and in other cases the strain recovery is achieved by heating the sample (thermoelastic effect). In many of these systems, partial softening of anomalous phonon modes has been reported as one approaches $M_{s}{ }^{4-8}$ However, the role of such premartensitic behavior in first-order displacive phase transitions remains controversial. ${ }^{9}$

To clarify this problem, we use a simple model to study the effects of phonon anomalies and anharmonicity on the behavior of the parent phase under applied shear stress. We find that the anomalous phonon behavior is closely connected with the pseudoelastic and thermoelastic behavior of the parent crystals just above $M_{s} .{ }^{10}$ Our results indicate that the anomalous elastic behavior observed in many martensitic systems is an intrinsic property of the parent crystal lattice near $M_{s}$, as opposed to usual materials in which the mechanical properties beyond the elastic limit are determined by dislocations and defects. We focused our attention on the Ni-Al alloys which are well-studied martensitic systems ${ }^{11,12}$ for which there exist recent detailed studies ${ }^{4,5}$ of anomalous phonon behavior in correlation with the martensitic transformation (see Fig. 1). As the temperature is lowered towards $M_{s}$, a dip appears in the dispersion curve near $q=0.16(1,1,0) 2 \pi / a$. At the same time, quasielastic scattering can be detected which can be connected with electron-microscopy studies ${ }^{5,13}$ indicating the existence of a modulating strain in the host crystal.

To model the elastic behavior of $\mathrm{Ni}-\mathrm{Al}$, we focus our attention on the shear-and-shuffle displacements of (110) planes in the lattice along the [1 10$]$ direction: Such displacements are involved in the transformation from the $B 2$ structure to close-packed (e.g., $7 R, 3 R$ ) structures. $^{14}$ This degree of freedom also corresponds to atomic dis- placements for the anomalous $[\xi \xi 0]$ transverse-acoustic (TA) phonon branch. Thus, we view the crystal as a collection of rigid (110) planes interacting with each other through anharmonic interactions. The values of the interplanar force constants for small displacements are chosen to reproduce the dispersion of the TA phonon. Because of the sharp features introduced by the phonon anomalies, we found that it is necessary in some cases to include interactions up to eight neighboring planes to get a good fit to the experimental phonon curves.

Since we expect large shear displacements to occur in a martensitic transformation, we have to include anharmonic effects in our model. This is done by imposing the condition of translation periodicity on each of the interplanar springs. Thus, we represent the total energy of the crystal as a sum of the effective interaction between

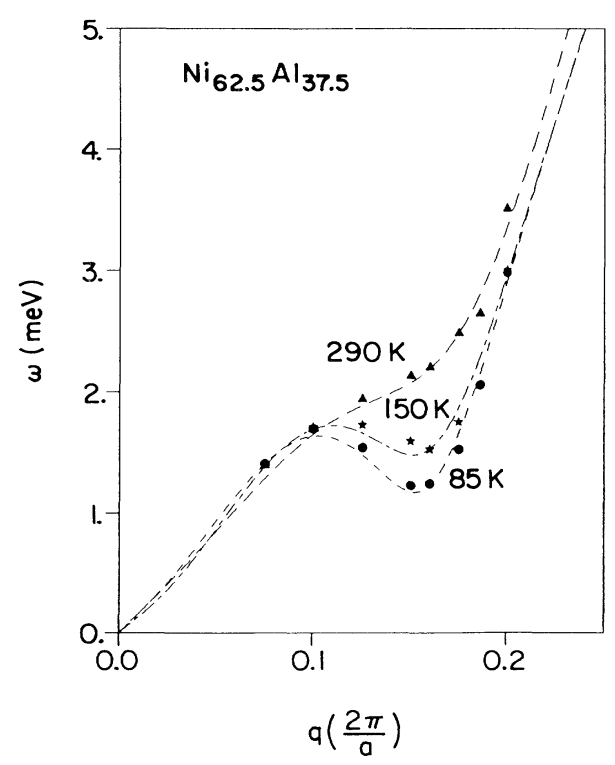

FIG. 1. Phonon-dispersion curves for the (110) TA model of $\mathrm{Ni}_{62.5} \mathrm{Al}_{37.5}$. The circles, stars, and triangles are experimentally measured frequencies at three temperatures, and the lines are theoretical fits with our model [Eq. (2) in the text]. 
individual (110) planes,

$$
E=-\sum_{n, p \neq 0} W_{p} \cos \left[(2 \pi / b)\left(X_{n}-X_{n-p}\right)\right],
$$

where $X_{n}-X_{n-p}$ measures the relative shear displacements (in the [1ㅣㅣ direction) of the $n$th and the $(n-p)$ th (110) planes and $b$ is the distance by which the system repeats itself when displaced in the shear direction. The index $n$ runs through the number of (110) planes in the crystal, while $p$ runs up to the range of interaction. The cosine form of the interplanar interaction satisfies the condition of translational periodicity.

In the presence of a TA phonon of wave vector $q$, the displacements of the planes are given by $X_{n}=X_{0} e^{i q n a}$, where $a$ is the interplanar separation. Expanding Eq. (1) to second order in $X_{0}$ yields the following Born-von Kármán expression for the phonon dispersion:

$$
\omega(q)^{2}=2 \sum_{p>0}\left[\frac{W_{p}}{M}\left(\frac{2 \pi}{b}\right)^{2}\right][1-\cos (q p a)] .
$$

The coupling constants $W_{p}$ 's are determined by fitting to experimental dispersion curves of the [ $\xi \xi 0]$ TA phonon branch in the $\mathrm{Ni}_{62.5} \mathrm{Al}_{37.5}$ alloy. Since the phonon spectra have been measured at three different temperatures $(85,150$, and $290 \mathrm{~K})$, we get three sets of $W_{p}$ 's, each giving the effective interactions at the corresponding temperature. Equation (1) is the simplest model that both satisfies the requirements of periodicity and reproduces the anomalous phonon dispersion.

The stress-strain (SS) behavior of a model system of 100 layers is then studied numerically as follows: We begin with the ground-state configuration $\left(X_{n}=0\right.$ for all $n$ ), corresponding to an ideal stacking of (110) planes in the $B 2$ structure. Small (equal and opposite) displacements are then imposed on the boundary layers, and the other layers are relaxed by a steepest-descent algorithm until the forces acting on them are zero. When equilibrium is achieved, the displacements of the boundary layers (strain), the forces at the boundary layers required to maintain the strain (stress), the energy, and the configuration of the system are all recorded. The displacements of the boundary layers are then increased by a small amount, and starting from the configuration of the previous equilibrium, we search again for the new equilibrium. Repeating this process, the "loading" part of the SS curves in Figs. 2(a)-2(c) are obtained for effective interactions corresponding to three different temperatures. During "unloading" the imposed strain is released gradually in small decrements. After each small decrement, the system is relaxed to equilibrium with the end layers position fixed. The simulations are repeated with a 200-layer system. Changes are negligible.

The loading SS curves are qualitatively similar for the three temperatures and have three regimes. Initially, stress increases almost linearly with strain. An examina-

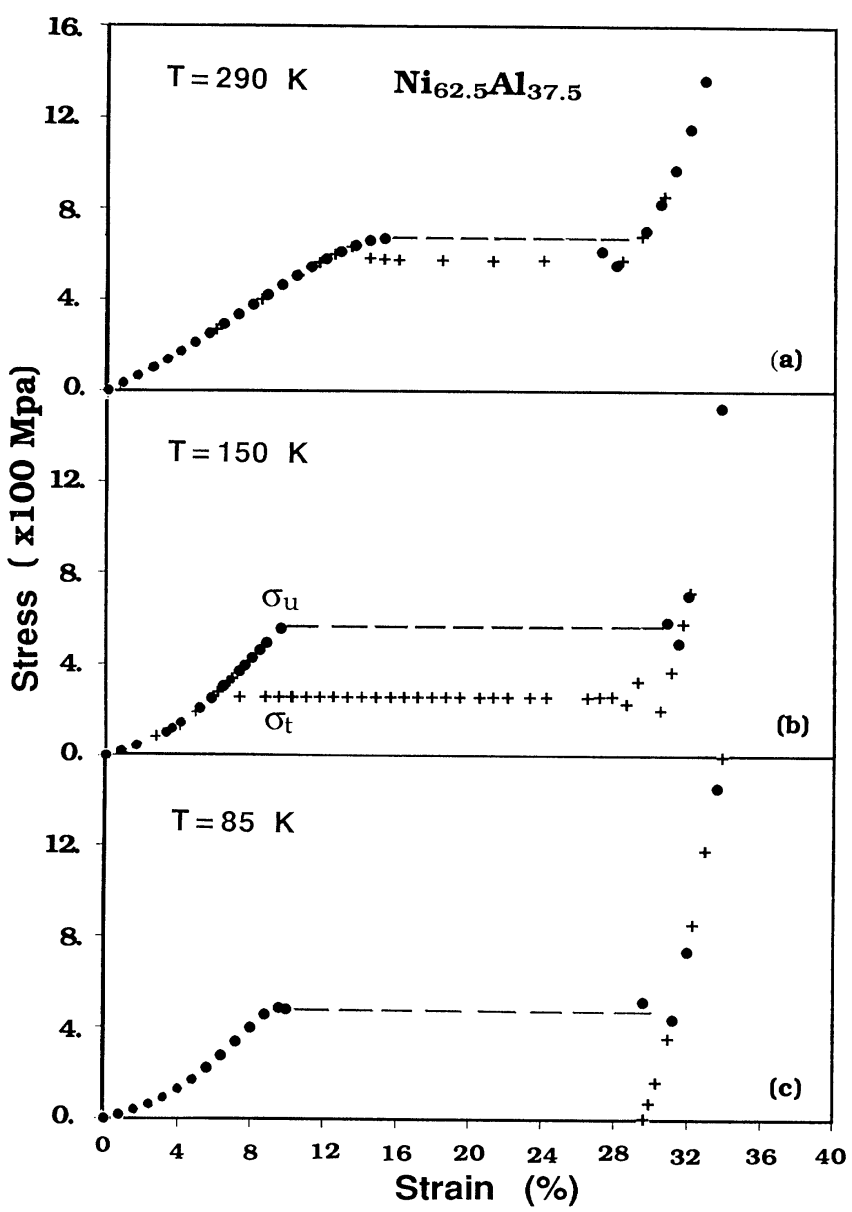

FIG. 2. Theoretical stress-strain (SS) relationships at three temperatures. Circles and crosses corresponds to "loading" and "unloading," respectively. The value of $\sigma_{u}$ depends on boundary conditions and simulation algorithms. In our simulation, the loading SS curves follow $\sigma_{t}$ once the $M$ phase is nucleated. In experimental situations, with constant external applied stress, the SS curves should follow $\sigma_{u}$ (dashed lines), leading to hysteresis in the transformation region.

tion of the configurations reveals a system with almost uniform homogeneous strain. When the stress increases to a critical value $\sigma_{u}$, which is higher for higher temperatures, the system segregates into two phases, one with a large strain (hereafter referred to as the $M$ phase) and the other with a smaller strain. The presence of a metastable large strain phase is apparent when we examine the energy change of the model system as a function of uniform strain (Fig. 3). At low temperatures, the energy-versus-strain ( $E-S)$ curve has two minima. At small stress, the elastic properties are governed by the curvature of the $E-S$ curve near the lower minimum ( $B 2$ phase). When the stress is sufficiently large, a stressinduced transformation between the $B 2$ and $M$ phases occurs, giving rise to the flat region in Fig. 2. In our simulation, once the $M$ phase is nucleated, the equilibri- 


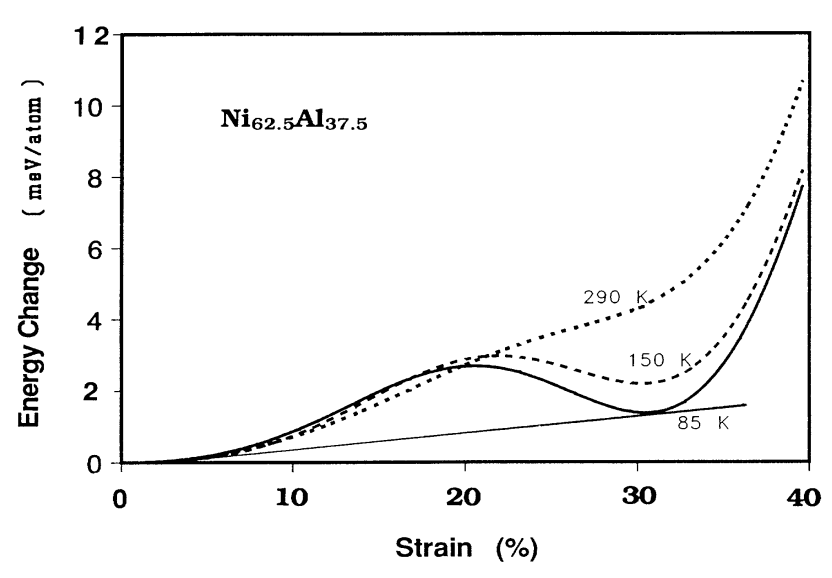

FIG. 3. Strain energies of our model Ni-Al system under uniform shear strain. The tangent lines give the transformation stresses in Fig. 2.

um stress drops to a lower strain-independent value $\left(\sigma_{t}\right)$. The increase of strain at constant stress is achieved by increasing the fraction of the $M$ phase. When the system is completely transformed to the $M$ phase, stress again increases with strain. In experimental situations, the strain of the system is measured with increasing load. Thus, once the loading stress reaches $\sigma_{u}$, the system will not be in mechanical equilibrium until the whole sample has transformed: There will be a sudden sharp increase in the strain and the actual shape of the hysteresis curves may depend on the physical conditions under which the experiment is performed. We have also performed molecular-dynamics simulations for our model under an increasing applied stress and verified this behavior. ${ }^{15}$ This shows that the results we obtained are not artifacts of the simulation algorithm chosen.

The unloading part of the SS curve varies qualitatively with temperature. For $T=290 \mathrm{~K}$ [Fig. 2(a)], pseudoelastic behavior is observed: On unloading, the system returns completely to the initial state although it is strained beyond the elastic limit. For $T=85 \mathrm{~K}$ [Fig. 2 (c)], a finite residual strain remains when the stress is released. The system settles into a metastable configuration with the stress-induced $M$-phase geometry. However, further relaxation with effective force constants corresponding to those of higher temperatures causes the system to return to its original state. Thus, complete strain recovery can be obtained by heating the system to higher temperatures (thermoelastic behavior). The SS curves from our simple model reproduce all the detailed features observed in experimental stress-loading measurements for many martensitic materials displaying the shape-memory effect. ${ }^{16}$ The occurrence of these anomalous elastic effects is closely associated with anomalous phonon dispersion: We have repeated our simulations with interaction parameters which are fitted to phonon-dispersion curves with the anomalous dip re- moved. Pseudoelastic behavior is not observed in these systems.

The transformation stress $\sigma_{t}$ we found numerically in Fig. 2 agrees well with the stress as found from the "common-tangent construction" on the $E-S$ curve in Fig. 3. Characteristic of the first-order nature of the transformation, the upper yield stress $\sigma_{u}$ is bigger than $\sigma_{t}$. The value of $\sigma_{u}$ depends on how easily the $M$ phase nucleates out of the parent phase under external stress, and so it is sensitive to the boundary conditions (it is at the boundary that homogeneity is broken which facilitates nucleation) and also depends on the simulation algorithm. The results shown in Fig. 2 are obtained with periodic boundary conditions. Simulations with free boundaries always lower $\sigma_{u}(12 \%$ for $150 \mathrm{~K})$. For the unloading part, the value of the transformation stress is found to be independent of boundary conditions. Moreover, the temperature at which the strain-induced state ceases to be metastable is found to be sensitive to boundary conditions. Thus, at the intermediate temperature, we obtain a residual strain on releasing the system with the free-boundary system, whereas with periodic boundary conditions, full strain recovery is obtained. The results for 85 and $295 \mathrm{~K}$ are independent of boundary conditions except for small details at the beginning and end of the constant-stress plateau. This further indicates that in our present model, nucleation of new phases occurs at the boundary.

To study the effects of thermal fluctuations, we have repeated the above calculations adding random displacements to the layers at various stages in our simulations. The sizes of the random displacements were chosen such that the total energy of the system is increased by an amount equal to $\frac{1}{2} k_{B} T$ per atom. We found that thermal fluctuations have negligible effects on our results, except by changing $\sigma_{u}$ by a few percent.

In normal materials, plastic deformation beyond the elastic limit occurs through the movement of dislocations. Such deformations are irrecoverable. Since our model does not take into account this deformation mechanism, the behavior described by our model will occur only when the upper yield stress $\left(\sigma_{u}\right)$ lies below the critical flow stress for dislocations in the material. However, for cases where $\sigma_{u}$ approaches zero as the temperature approaches $M_{s}$, there exists a temperature range above $M_{s}$ where the pseudoelastic and thermoelastic effects described above can be observed.

Within our simple model, the $M$-phase geometry occurs at a strain close to that expected for the transformation to a close-packed $3 R$ phase. The configuration of the strain-induced phase in our model corresponds to a $3 R$-like structure, whereas a $7 R$ structure has been reported for $\mathrm{Ni}-\mathrm{Al}$ alloys with $\mathrm{Ni}$ concentration less than 63\%. ${ }^{13,17}$ A possible reason for this discrepancy is that the assumption of a single cosine term in Eq. (1) might be too simple to describe the system. Higher terms can 
be included if extra information is available (e.g., the change in the phonon-dispersion curves with stress or additional information from first-principles calculations). Moreover, since both the $3 R$ and $7 R$ are close-packed structures, they have similar energies and which structure one obtains might depend on how the stress is applied. ${ }^{17}$ The change in the shear-induced structure would change the range of strains for the stress plateau; for example, a $7 R$ structure would give a strain near $14 \%$ for the stress-induced martensite. Another oversimplification in our model is the neglect of other strains which in the real crystal act in cooperation with the (110) shear to form the martensitic phase. This leads to an overestimate of the transformation stress in our model.

In summary, we have shown that a simple onedimensional model can reproduce the pseudoelastic and thermoelastic behaviors at a temperature range just above $M_{s}$ and elucidate the underlying physics of these exotic phenomena. We also note that the phonon anomaly in $\mathrm{Ni}-\mathrm{Al}$ is a consequence of strong electron-phonon interaction and Fermi surface nesting. ${ }^{18}$

We thank Professor B. N. Harmon for stimulating discussions. Ames Laboratory is operated for the U.S. Department of Energy by Iowa State University under Contract No. W-7405-ENG-82. This investigation was supported by the Direction for Energy Research, Office of Basic Energy Sciences.

\footnotetext{
(a) Permanent address: Physics Department, Wuhan University, Wuhan, People's Republic of China.

'Shape Memory Effects in Alloys, edited by J. Perkins (Plenum, New York, 1975).

${ }^{2}$ Shape Memory Materials, edited by K. Otsuka, K. Shimizu, M. Doyama, S. Somiya, and R. P. H. Chang (Materials Research Society, Pittsburgh, 1989).

${ }^{3}$ See reviews by K. Otsuka and K. Shimizu, Int. Met. Rev.
}

31, 93 (1986); Met. Forum 4, 142 (1981).

${ }^{4}$ S. M. Shapiro, B. X. Yang, G. Shirane, Y. Noda, and L. E. Tanner, Phys. Rev. Lett. 62, 1298 (1989).

${ }^{5}$ S. M. Shapiro, J. Z. Larese, Y. Noda, S. C. Moss, and L. E. Tanner, Phys. Rev. Lett. 57, 3199 (1986).

${ }^{6}$ S. K. Satija, S. M. Shapiro, M. B. Salamon, and C. M. Wayman, Phys. Rev. B 29, 6031 (1984); H. Teitze, M. Mullner, and B. Renker, J. Phys. C 17, L529 (1989).

${ }^{7}$ M. Mori, Y. Yamada, and G. Shirane, Solid State Commun. 17, 127 (1975).

${ }^{8}$ See review article by N. Nakanishi, Prog. Mater. Sci. 24, 143 (1980).

${ }^{9}$ Proceedings of the Workshop on First-Order Displacive Phase Transformations, Oakland, California, October 1988 [Mat. Sci. Eng. A 127 (1990)].

${ }^{10}$ Pseudoelastic behavior can also be observed for some cases when the crystal is in the martensitic phase below $M_{s}$. Such behavior originates from the movement of twin boundaries [see Ref. 3 and A. Zangwill and R. Bruinsma, Phys. Rev. Lett. 53, 1073 (1984)]. However, in this paper we focus on the temperature region above $M_{s}$ where pseudoelastic behavior is caused by stress-induced transformation rather than twin boundary movements.

${ }^{11}$ K. Enami and S. Nenno, Metall. Trans. 2, 1487 (1971).

${ }^{12}$ Y. K. Au and C. M. Wayman, Scripta Metall. 6, 1209 (1972).

${ }^{13}$ D. Schryvers, L. E. Tanner, and S. M. Shapiro, in Shape Memory Materials (Ref. 2), p. 35; D. Schryvers, L. E. Tanner, and G. Van Tendeloo, in Proceedings of the NATO Advanced Study Institute on Phase Stability, Crete, edited by A. Gonis and M. Stocks (Martinus Nijhoff, The Netherlands, 1988).

${ }^{14}$ These transformations are accompanied by other distortions, e.g., Bain strain, that serve to alter in-plane atomic arrangements.

${ }^{15}$ Y. Y. Ye, C. Z. Wang, C. T. Chan, and K. M. Ho (to be published).

${ }^{16}$ See, e.g., L. A. Shapard, in Shape Memory Effects in Alloys (Ref. 1), p. 419.

${ }^{17}$ V. V. Martynov, K. Enami, L. G. Khandros, S. Nenno, and A. V. Tkachanko, Phys. Met. Metall. 55, 136 (1983).

${ }^{18}$ G. L. Zhao and B. N. Harmon (to be published). 